\title{
PAH emission variations within the resolved starbursts of NGC 253 and NGC $1808^{\star}$
}

\author{
L. E. Tacconi-Garman ${ }^{1}$, E. Sturm² ${ }^{2}$ M. Lehnert ${ }^{2}$, D. Lutz², R. I. Davies², and A. F. M. Moorwood ${ }^{1}$ \\ ${ }^{1}$ European Southern Observatory, Karl-Schwarzschild-Strasse 2, 85748 Garching, Germany \\ e-mail: 1tacconi@eso.org \\ 2 Max-Planck-Institut für extraterrestrische Physik, Postfach 1312, 85741 Garching, Germany
}

Received 25 November 2003 / Accepted 4 November 2004

\begin{abstract}
In order to better characterise the usefulness of PAH emission as a tracer of star formation, we have undertaken a programme of $3 \mu \mathrm{m}$ narrowband imaging of a sample of nearby template galaxies covering a wide range of metallicity, star formation activity, and nuclear activity. In the present paper we present first results of this programme: high spatial resolution images of PAH feature emission and the adjacent continuum emission from the central regions of the nearby starburst galaxies NGC 253 and NGC 1808, taken with ISAAC at the VLT-UT1. Globally, the feature emission is seen to peak on the central starburst regions of both sources. On smaller scales, however, we see no general spatial correlation or anti-correlation between the PAH feature emission and the location of sites of recent star formation, suggesting that the degree to which PAH feature emission traces starburst activity is more complicated than previously hypothesized based on results from data with lower spatial resolution. We do find spatial correlations, though, when we consider the feature-to-continuum ratio, which is low at the positions of known super star clusters in NGC 1808 as well as at the position of the IR peak in NGC 253. We take this to imply a decrease in the efficiency of PAH emission induced by the star formation, caused either by mechanical energy input into the ISM, photoionisation of the PAH molecules, or photodissociation of the PAH molecules. All three hypotheses are discussed. In addition, for the first time we present observations of PAH feature emission in the superwind of a starbursting system (NGC 253), providing strong support that winds are heavily mass-loaded and entrain substantial amounts of ambient ISM. We have also found a plausible connection between observed $\mathrm{NaD}$ absorption, $\mathrm{H}_{2}$, and PAHs above the plane of NGC 253. This observation has important implications for enriching galaxy halos and possibly the intergalactic medium with small dust grains.
\end{abstract}

Key words. infrared: ISM - ISM: lines and bands - infrared: galaxies - galaxies: starburst - galaxies: individual: NGC 253 galaxies: individual: NGC 1808

\section{Introduction}

The mid-infrared emission features at 3.3, 6.2, 7.7, 8.6, 11.3 and $12.7 \mu \mathrm{m}$, ascribed to aromatic carriers that are transiently excited by single (UV) photons, are a powerful diagnostic of the conditions in dusty external galaxies. About ten percent of the bolometric luminosity of a galaxy can be radiated in these features, making their observation possible even for faint and distant galaxies. In the following we will call them "PAH" (polycyclic aromatic hydrocarbons) according to one of the most popular designations. Building on previous groundbased work (Roche et al. 1991; Moorwood 1986), studies of extragalactic PAH emission have been extended considerably through low resolution spectroscopy with the Infrared Space Observatory. For example, Genzel et al. (1998), Lutz et al. (1998), Rigopoulou et al. (1999), and Tran et al. (2001) have used the PAH strength (relative to the continuum) as a tool to quantitatively disentangle the contributions from

^ Based on observations made with ESO Telescopes at the Paranal Observatory under programme ID 68.B-0264(A). starbursts and AGNs to the energy budget of dusty infrared bright galaxies (starbursts, AGNs and ULIRGs). Laurent et al. $(2000,2001)$ have developed a diagnostic that further discriminates between an AGN and starburst origin of the continuum proper on the basis of its slope. The main observational facts on which these analyses are based are the following:

First, PAH emission is strong and ubiquitous in normal and starbursting galaxies, with little variation in shape and strength from source to source (Helou et al. 2000; Rigopoulou et al. 1999). While observations in our galaxy show a clear decrease in PAH emission when approaching a hot star, and when going inward from PDRs to HII regions, (e.g. Boulanger et al. 1988; Verstraete et al. 1996), these variations largely average out on a galactic scale making PAH emission a valuable tracer of stellar (soft UV) emission.

Second, PAH emission is weak (or has a small equivalent width) close to a strong AGN, as indicated by mapping at a resolution of $\sim 5^{\prime \prime}$ (Mirabel et al. 1999; Le Floc'h et al. 2001). Note, however, that this resolution is not high enough to distinguish between dilution of PAHs by a warm continuum from a 
nuclear (point) source on the one hand (Moorwood 1999) and destruction of the PAHs on the other hand. Close to the nucleus the PAHs are most likely destroyed by unshielded EUV/X-ray radiation of the AGN (Voit 1992). PAH emission is often detected on larger scales in AGN hosts (Clavel 2000).

Third, PAH emission is fainter in low metallicity galaxies (e.g. Madden et al. 2000; Contursi et al. 2000).

Investigators using current and future infrared missions are making and will continue to make extensive use of the PAHs as a tool to study infrared bright galaxies at high redshifts and, e.g., constrain the contribution of star formation and AGN to the cosmic IR background (e.g. Devost et al. 2004; Armus et al. 2004; Spoon et al. 2004; Smith et al. 2004; and Houck et al. 2004). Application of the locally developed PAH diagnostics is, however, subject to the caveat that bright high redshift sources may have lower metallicities and higher star formation densities. This provides further strong motivation to address a number of questions left open by global studies of nearby galaxies.

For instance: what is the effect of extremely high star formation density on the PAH properties? Do PDRs and HII regions still "average out" in the same way over the entire galaxy? Is there a PAH deficiency in the same way and for similar reasons as the "[C II] deficiency" observed in ULIRGS, and likely related to the high intensity of the UV radiation? Observations of ULIRGs indicate a deficiency of at least a factor of 2 in relative PAH luminosity (Rigopoulou et al. 1999; Luhman et al. 2003), but these global measurements cannot satisfactorily discriminate among the effects of star formation density, extinction, or AGN contributions. A related question is whether the weakness of PAHs in low metallicity dwarfs is entirely an abundance effect or also related to much of their star formation occurring in compact super star clusters and in their possible higher UV intensities per unit star formation.

High spatial resolution PAH imaging of nearby starburst and active galaxies will help in answering these questions. Cryogenic space telescopes with $60-80 \mathrm{~cm}$ mirrors like ISO and Spitzer, observing the 6-13 $\mu \mathrm{m}$ PAHs lack the required (sub)arcsecond spatial resolution, and published groundbased data are restricted to a handful of sources (NGC 5128 (Turner et al. 1992), NGC 253 (Kalas \& Wynn-Williams 1994), NGC 7469 (Mazzarella et al. 1994), M 82 (Normand et al. 1995; Satyapal et al. 1995), and Mrk 231 (Harvey et al. 1999)), all of which suffer from poor spatial resolution and/or low $\mathrm{S} / \mathrm{N}$ and/or low depth. With this in mind we have started a programme to obtain seeing-limited PAH images of a small but meaningful sample of nearby galaxies of different types, well studied Seyferts, dusty starbursts and low metallicity starbursts. Here we present first results on the two starburst galaxies NGC 253 and NGC 1808.

\section{The targets}

At a distance of $2.5 \mathrm{Mpc}$ (Forbes et al. 2000), NGC 253 is among the nearest and best-studied starburst galaxies. However, its relatively high inclination and central obscuration has led to difficulties in establishing relative astrometries between observations at differing wavelengths and interpreting the nature of the observed clumpy structure. We will not use the data in this paper to address the astrometric issues, but rather adopt the astrometry presented in the comprehensive work of Forbes et al. (2000). In that work it is demonstrated that a number of discrete sources observed at wavelengths from the optical to the millimetre lie in an elliptical ring of radius $\sim 50 \mathrm{pc}$. The dominant feature in images at wavelengths from $1.6 \mu \mathrm{m}$ to $20 \mu \mathrm{m}$ (Sams et al. 1994; Kalas \& Wynn-Williams 1994; Keto et al. 1999; Forbes et al. 2000; A. Gilbert, private communication) lies in that ring. The radio nucleus lies interior to this ring, near its centre (Forbes et al. 2000). The natures of the dominant IR source and the nucleus itself is addressed in Sects. 4.5 and 4.6, respectively. In addition to these (near-)nuclear features, NGC 253 has a prominent foreground (and less prominent background) starburst wind-blown bubble, seen in both $\mathrm{H} \alpha$ (Lehnert \& Heckman 1996) and X-rays (Pietsch et al. 2000; Strickland et al. 2000). In Sect. 4.4 we discuss the PAH feature emission associated with this bubble.

NGC 1808, lying at a distance of 10.9 Mpc (Tacconi-Garman et al. 1996, hereafter TGSE96), is a galaxy that certainly harbours a starbursting circumnuclear region (Collison et al. 1994; Krabbe et al. 1994; Kotilainen et al. 1996; TGSE96). Early evidence for the presence of an active nucleus in the form of extended wings on both $\mathrm{H} \alpha$ and [NII] line profiles was presented by Véron-Cetty \& Véron (1985), but this was later discounted by several authors (e.g. Phillips 1993). More recent possible evidence for an AGN comes from X-ray variability (Awaki et al. 1996), though those authors caution that the case for an AGN is still not clear.

In addition, NGC 1808 shows evidence for outflow from the nucleus (Phillips 1993), although without the edge-brightened $\mathrm{H} \alpha$ emission bubble as is seen in NGC 253. That this outflow is dusty is particularly easily seen in Fig. 2 of Phillips (1993). This dusty outflow could be driven by circumnuclear supernovae, evidence for which is seen in the radio continuum observations of Saikia et al. (1990) and Collison et al. (1994). These observations show that there are a dozen compact (unresolved) radio continuum knots in the circumnuclear region of NGC 1808. The spectral indices of these knots are consistent with a nonthermal, supernova, origin (Collison et al. 1994).

In addition to these radio continuum knots, NGC 1808 also exhibits conspicuous $K$-band knots, which are likely to be bound, young (6-8 Myr), super star clusters (TGSE96). These knots are spatially distinct from the radio continuum knots, which can be understood assuming that roughly half of the smoother $K$-band component in the circumnuclear region arises from red giants and supergiants between 10 and $500 \mathrm{Myr}$ old (TGSE96). The starburst is then seen as having a smooth component with particularly young knots superposed on it.

\section{Observation and data processing}

The data have been obtained with the infrared camera and spectrograph ISAAC on ESO's Very Large Telescope UT1 (ANTU) on 24/25 October 2001. ISAAC was operated in the LWI4 mode, with a pixel scale of 0.0709 arcsec/pixel, and a corresponding field of view of $73^{\prime \prime} \times 73^{\prime \prime}$. We used the two narrow band filters NB_3.28 and NB_3.21, centred on the $3.28 \mu \mathrm{m}$ PAH feature and the underlying continuum at $3.21 \mu \mathrm{m}$, 
respectively ${ }^{1}$. The spectrum near the $3.3 \mu \mathrm{m}$ PAH feature shows continuum on the blue side and a weaker PAH feature at $3.4 \mu \mathrm{m}$ (Sturm et al. 2000) making the use of a single filter for continuum more accurate than if the feature were bracketted with two filters.

In both filters we took a series of observations of the target itself and of sky positions (for the background subtraction), with some jitter applied to the individual positions. For NGC 253 sky information was obtained from exposures totally off source, while for NGC 1808 sky information was constructed from the observations with the target in alternating corners of the detector array. After frame selection the total on-target exposure time in the NB_3.28 filter was 34 min and $40 \mathrm{~min}$ for NGC 253 and NGC 1808, respectively. The weather conditions were quite unsettled, with seeing at $3.3 \mu \mathrm{m}$ between 0.4 and 0.7 arcsec and highly variable atmospheric transmission rendering photometric calibration unattainable. The data were reduced using standard techniques as outlined in the ISAAC Data Reduction Guide $1.5^{2}$, including pairwise subtraction of sky images from object images and registering the resulting images to a common position. For NGC 253 we have aligned the brightest peak in the ISAAC data with the position of the IR maximum (Forbes et al. 2000), itself an average of the positions found in Piña et al. (1992), Keto et al. (1993) and Kalas \& Wynn-Williams (1994). In the case of NGC 1808 we have aligned the dominant central emission peak with the nuclear peak seen in the $K$-band (TGSE96). Co-added NB_3.21 (continuum) images were then subtracted from the co-added NB_3.28 (PAH plus continuum) images to produce continuum-free $\mathrm{PAH}$ feature images.

\section{Results}

\subsection{General remarks}

The resulting images are shown in Figs. 1 and 2. In both of these figures the left panel is the $3.21 \mu \mathrm{m}$ continuum emission while the right panel shows the continuum-subtracted $3.28 \mu \mathrm{m}$ PAH feature emission. The continuum in both cases agrees very well with the continuum observed in the $K$-band (NGC 253: Sams et al. 1994; NGC 1808: TGSE96) suggesting that a large fraction of the continuum emission is due to starlight.

The distribution of the PAH feature emission from NGC 253 is similar to that of the continuum emission, including the increase toward the central starburst region, but with weak extensions/tails to the south and east of the main emission region with less prominent "counter" tails to the north and west.

The PAH feature emission from NGC 1808 also bears some resemblance to the continuum emission (e.g. both are bright

\footnotetext{
1 These filters are both $0.05 \mu \mathrm{m}$ wide and have very similar spectral responses relative to their central wavelengths. The overall instrumental response to a flat continuum source is the same for both filters. Further, the NB_3.28 filter is well matched to both the shape of the PAH feature and to the redshift of the sources considered here. Indeed, it is with these criteria in mind that the source selection was made.

2 http://www. eso.org/instruments/isaac/drg/html/ drg.html
}

in the inner starbursting region), though also with important differences. The prominent tail and arc toward the southeast of the nucleus seen in the feature emission is virtually absent in the continuum. In addition, although in both the continuum and feature emission maps the central bar-like structure is evident, it is only in the feature map that an equally prominent arc is seen to the east of the nucleus.

The differences in structure seen in the PAH feature emission and continuum maps for both sources are especially evident in the feature-to-continuum maps we have constructed from the data. To produce these maps we have clipped the $5 \times$ 5 boxcar-smoothed continuum-subtracted feature and continuum maps at their respective $3 \sigma$ levels before dividing. Thus, the feature-to-continuum maps only show values where both PAH feature and continuum surface brightnesses are significant. The resulting feature-to-continuum maps are shown in the bottom panels of Figs. 3 and 4.

We discuss our observed feature-to-continuum findings in more detail in the following subsections. Here we only note that while smallscale extinction variations may play a role in creating the observed structures of the PAH feature emission and the $3.3 \mu \mathrm{m}$ continuum, it is likely that most of such extinction is in the foreground of both the feature and continuum emission. Hence, the ratio is largely unaffected by such as yet unknown extinction variations.

\subsection{PAH emission on scales of the central starbursting regions}

In Figs. 3 and 4 we show our observed continuum-subtracted PAH feature emission maps (top) and the corresponding feature-to-continuum maps (bottom). With both galaxies we find that the PAH feature emission is seen to trace the region of strong star formation. In the case of NGC 253 this is clearly demonstrated by stronger PAH feature emission from the region with the highest concentration of compact radio sources (both HII regions and supernova remnants; Ulvestad \& Antonucci 1997). Moreover, we find an excellent morphological agreement between the Paschen $\alpha$ emission at HST resolution (Alonso-Herrero et al. 2003) and the bright inner PAH feature emission observed with ISAAC, as shown in Fig. $5^{3}$. Although to our knowledge no similar high resolution ionised hydrogen data exist for NGC 1808 we do find that the PAH feature emission is strong in the inner star-forming region which is peppered with knots of particularly compact star-forming regions, as traced by the $K$-band observations of TGSE96.

Although the PAH emission is seen to peak in the inner, starbursting, regions in these galaxies, the PAH featureto-continuum images (bottom panels of Figs. 3 and 4) reveal a general depression in that ratio in the inner regions of these systems. In both cases the feature-to-continuum ratio is lowest

\footnotetext{
${ }^{3}$ As we show in Sect. 4.4 there is also a strong morphological correspondence between the lower surface brightness PAH feature emission and the extended $\mathrm{H} \alpha$ emission. Thus the lack of $\mathrm{Pa} \alpha$ emission at the position of the extended PAH feature emission likely is due to the depth of the exposure at $\mathrm{Pa} \alpha$.
} 


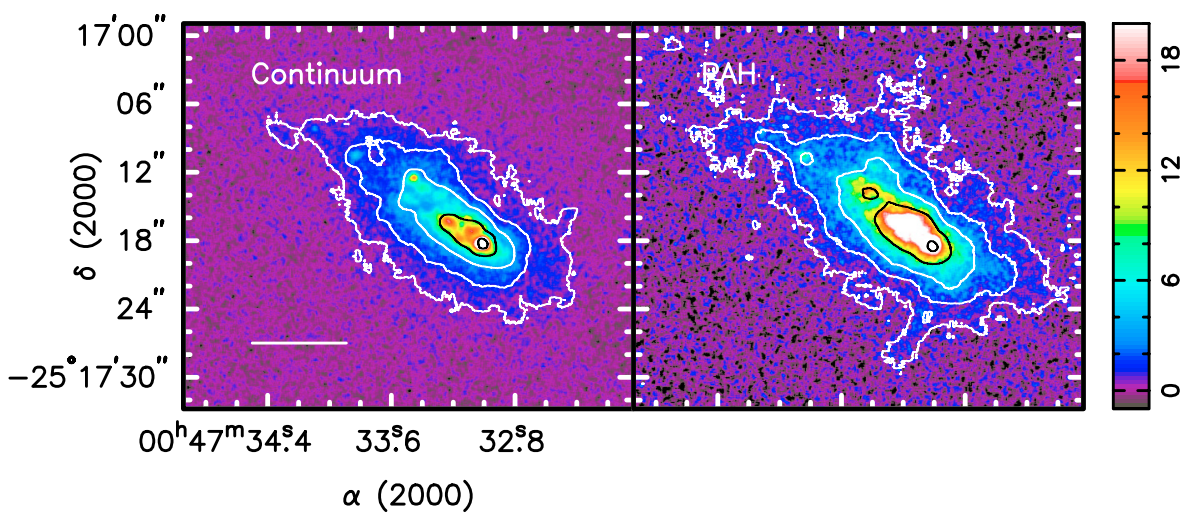

Fig. 1. $3.21 \mu \mathrm{m}$ continuum emission (left) and $3.28 \mu \mathrm{m}$ continuum-subtracted PAH feature emission (right) from NGC 253. The data have been spatially smoothed by a $5 \times 5$ boxcar, and the relative contours (based on data smoothed with a $20 \times 20$ boxcar) are logarithmically spaced at 2 , $5.2,13.4,34.7$, and $90 \%$ of the peak flux values (68 and 44 counts for the left and right images, respectively). The bar in the lower left corner of the left panel indicates $100 \mathrm{pc}$ at the distance to NGC 253 (2.5 Mpc, Forbes et al. 2000). The images have not been scaled to a common peak level. The wedge to the right shows relative surface brightness levels.

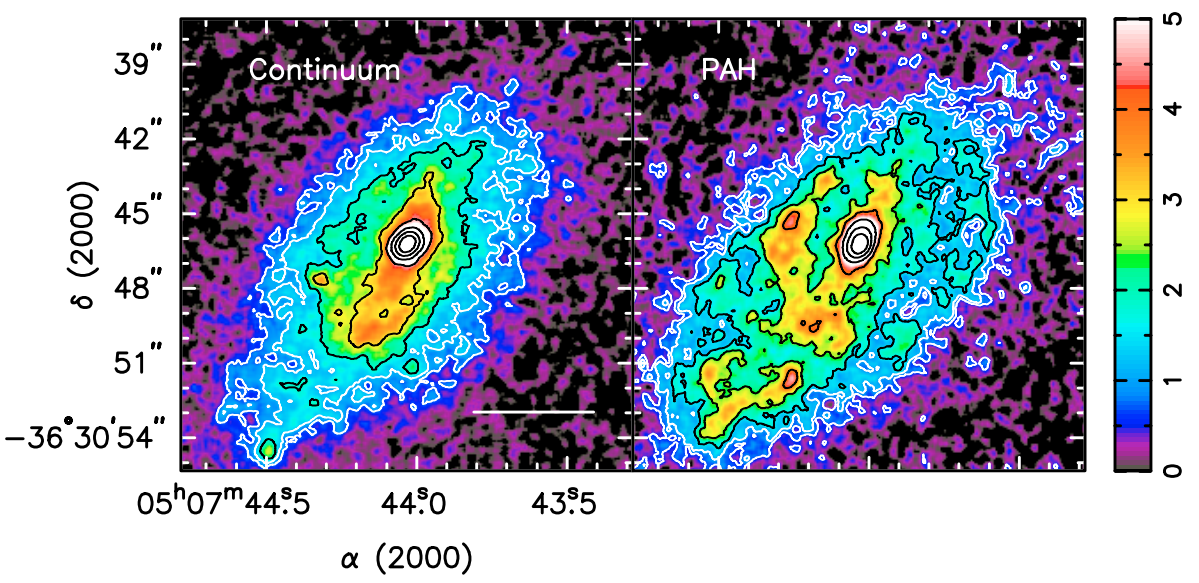

Fig. 2. $3.21 \mu \mathrm{m}$ continuum emission (left) and $3.28 \mu \mathrm{m}$ continuum-subtracted PAH feature emission (right) from NGC 1808. The data have been spatially smoothed by a $5 \times 5$ boxcar, and the relative contours are logarithmically spaced at $2.5,4.0,6.3, \ldots, 63 \%$ of the peak flux $(29$ and 24 counts for the left and right images, respectively). The bar in the lower right corner of the left panel indicates $250 \mathrm{pc}$ at the distance to NGC 1808 (10.9 Mpc (TGSE96)). The images have not been scaled to a common peak level. The wedge to the right shows relative surface brightness levels.

in the region with the brightest $K$-band emission ${ }^{4}$, suggesting a causal connection between the smooth starburst region and the depression of the feature-to-continuum ratio.

\subsection{PAH emission on scales of the super star clusters}

A detailed inspection of the bottom panels of Figs. 3 and 4 reveals that there is a tendency for the feature-to-continuum ratio to be lower at the position of the IR peak in NGC 253, and at the positions of the $K$-band knots in NGC 1808 . A prime example of this is the feature-to-continuum in the region of the $K$-band knot in NGC 1808 lying at $\alpha_{2000}=05^{\mathrm{h}} 07^{\mathrm{m}} 44.44$, $\delta_{2000}=-36^{\circ} 30^{\prime} 51.9^{\prime \prime}$. The location of that knot is a local minimum of the ratio, which is surrounded by an almost complete ring where the ratio is far higher. A similar, almost perfectly

\footnotetext{
${ }^{4}$ In the interest of clarity, we have not shown $K$-band contours in Fig. 4. For these contours the reader is referred to Fig. 2 (right) of TGSE96.
}

circular ring is present around the IR peak in NGC 253. Other less prominent examples are shown in the blow-ups in Fig. 6.

In order to better quantify this visual impression, we have computed radial profiles of the PAH feature emission, $3.3 \mu \mathrm{m}$ continuum emission, and the $\mathrm{PAH}$ feature-to-continuum ratio around each of the $27 \mathrm{~K}$-band knots identified in TGSE96 as having strongly peaked $K$-band emission. We have considered only those sources for which the maximum aperture used $\left(0.5^{\prime \prime}\right.$ radius) is completely contained in our feature-to-continuum map (knots 2, 3, 6, 10, 12, 13 (the nucleus), 14-17, 19-21, 23, 24, and 26 using the numbering scheme of TGSE96). Most of the individual radial profiles indicate that the location of the $K$-band knots corresponds to ratio minima, but to demonstrate that this is true for the ensemble we have computed a luminosity-weighted mean profile which is shown in Fig. 7. In order to avoid strongly biasing the result to that of the much brighter nucleus we have made separate radial profiles for the nucleus (only) and for the ensemble of knots excluding the nucleus. It is clear from that figure that the 


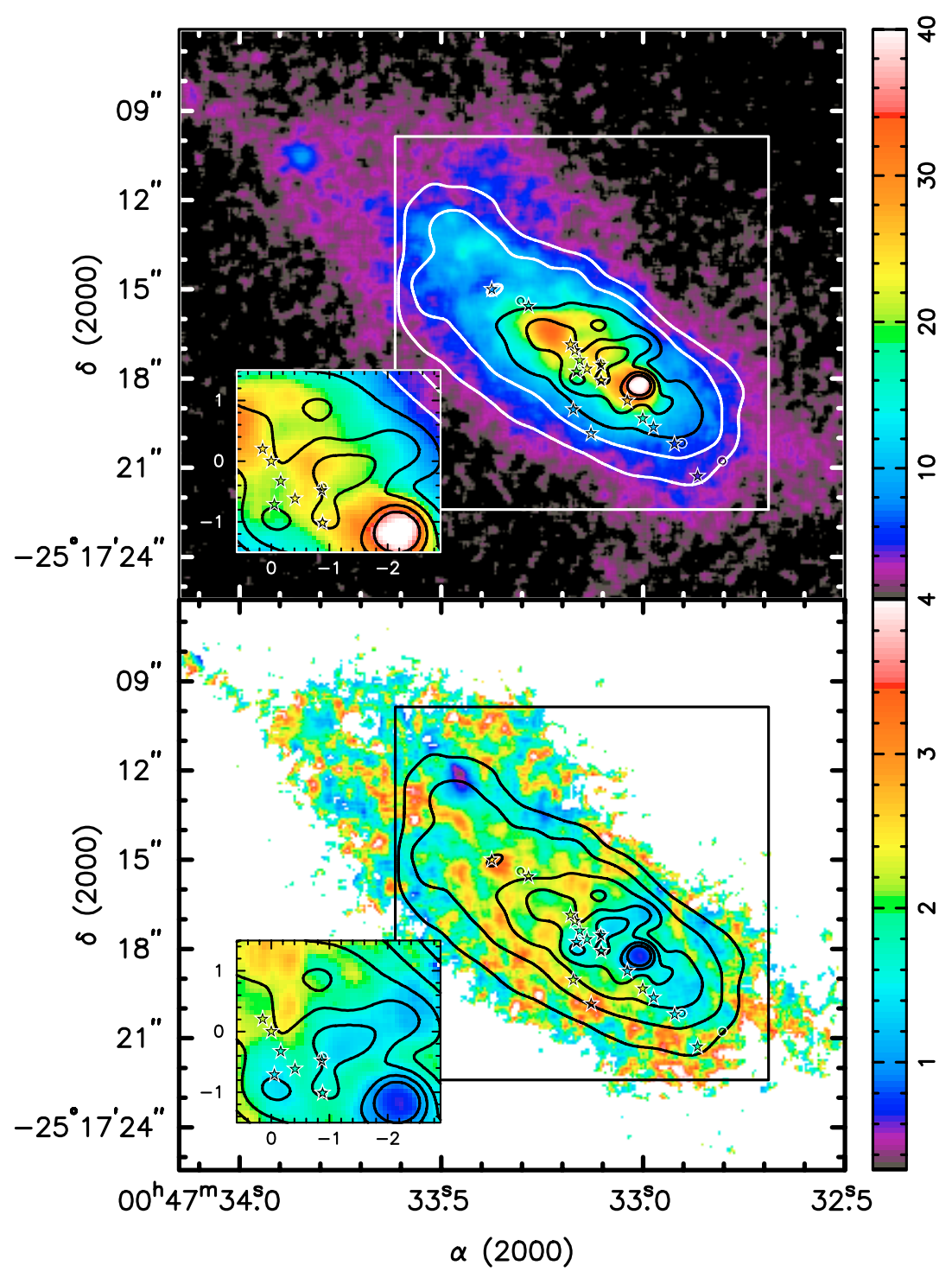

Fig. 3. The top panel shows relative contiunuum-subtracted PAH feature emission in the central regions of NGC 253 based on our observational results; the wedge to the right indicates relative surface brightnesses in the image. In the bottom panel we show the feature-to-continuum ratio over the same area of NGC 253; the wedge to the right indicates feature-to-continuum values. Contours of the $K$-band flux (Sams et al. 1994, with the peak position shifted to agree with that of Forbes et al. 2000) are shown at 6,9,14,21,33, and 50\% of the peak $K$-band flux. The thin square represents the field-of-view of those $K$-band observations. Circles represent ultracompact HII regions from Johnson et al. (2001; shifted onto the reference frame of Ulvestad \& Antonucci 1997). Stars show the locations of $2 \mathrm{~cm}$ radio continuum sources (both thermal and non-thermal) from Ulvestad \& Antonucci (1997). Finally, we provide for clarity blowups of the circumnuclear region in the lower left corners of each panel, where in each case the box is labelled with offsets from the nucleus in arcseconds.

$3.3 \mu \mathrm{m}$ continuum emission is also peaked at the position of the $K$-band knots, lending support for the stellar origin of the $3.3 \mu \mathrm{m}$ continuum. Further the regions around these young, super star clusters exhibit a paucity of PAH emission relative to the continuum only.

For comparison with NGC 1808 we have computed the radial profiles of $\mathrm{PAH}$ emission, continuum emission, and feature-to-continuum ratio centred on the IR peak of NGC 253. To make the comparison fair we have used the same set of annuli as for NGC 1808, scaled by the ratio of the distances to the two galaxies such that we are plotting the same physical regions in both cases. Our resulting radial profiles are shown in the last column of Fig. 7. Here we see the same depression in the feature-to-continuum ratio at the position of the IR peak, though even more extreme than is the case for NGC 1808.

\subsection{PAH emission from the superwind in NGC 253}

Extensions and tails are seen in the PAH emission image (Fig. 1, right), most obviously to the south of the nucleus. They are not present, or very weak in the continuum image (Fig. 1, left). For that reason they are not included in the feature-to-continuum map shown in Fig. 3, despite a feature-to-continuum ratio which is clearly high $(\geq 4-5)$. 


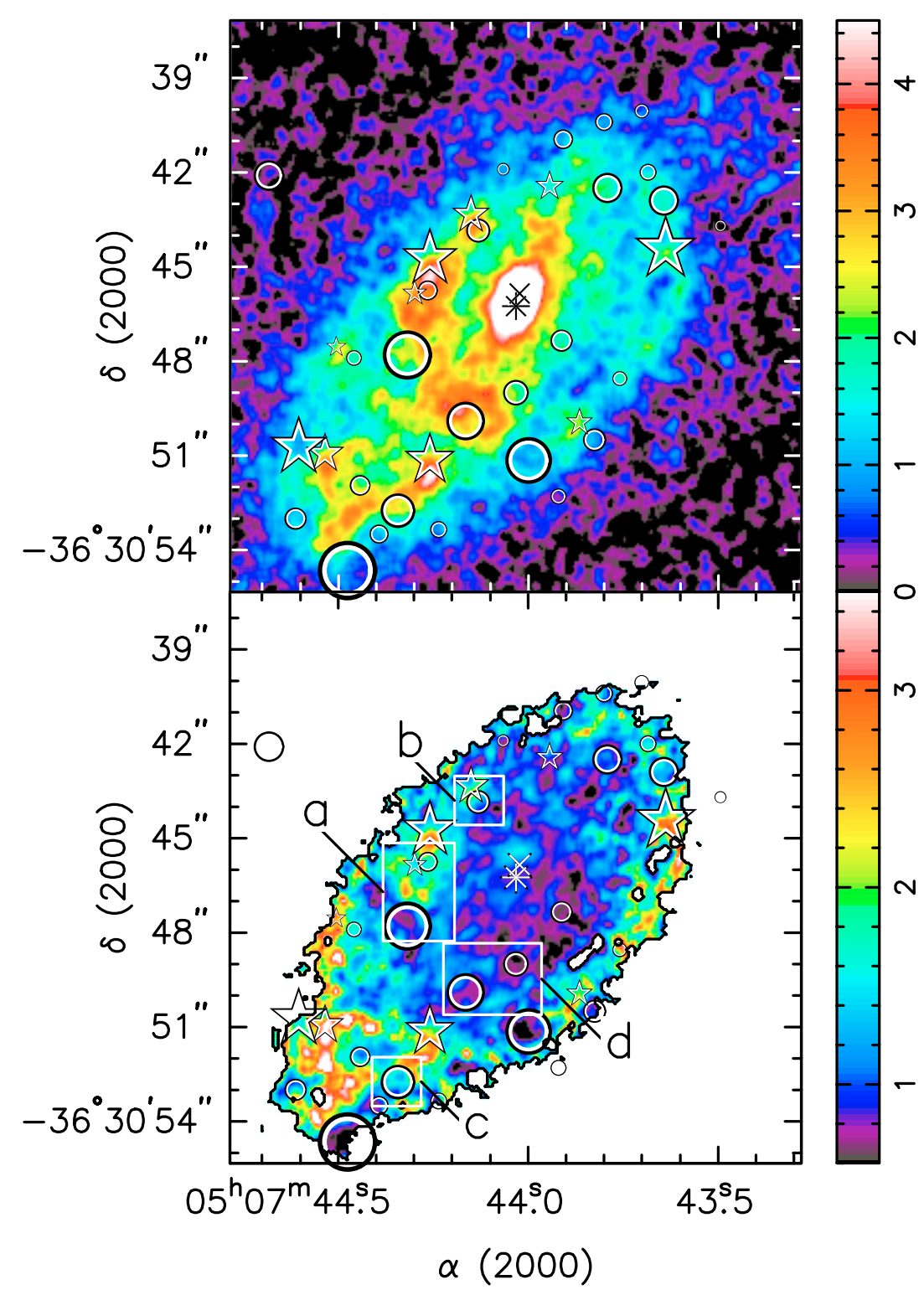

Fig. 4. The top panel shows relative continuum-subtracted PAH feature emission in NGC 1808 based on our observational results; the wedge to the right indicates relative surface brightnesses in the image. In the bottom panel we show the feature-to-continuum ratio over the same area; the wedge to the right indicates feature-to-continuum values. Shown also are circles showing the locations of the $K$-band knots reported by Tacconi-Garman et al. (1996), scaled by their derived $K$-band luminosity (for clarity the position of the nucleus is indicated by a simple + ). In addition here we show as stars the locations of the $3.6 \mathrm{~cm}$ radio continuum knots (Collison et al. 1994), scaled by their peak flux density (for clarity the positions of their knots $\mathrm{A} 3$ and $\mathrm{O}$ (the nucleus) are indicated by $\times$ symbols). In the bottom panel, the thick black contour serves to distinguish those white regions which have high feature-to-continuum values from the background where that ratio could not be determined. Labelled boxes in that panel show those regions illustrated in Fig. 6.

The appearance of these faint PAH emission features perhaps suggests outflowing PAH dust structures. NGC 253 does have a well-known supernova-driven wind, observed in $\mathrm{H} \alpha$ (Lehnert \& Heckman 1996) as well as in the X-ray (Pietsch et al. 2000; Strickland et al. 2000). A comparison between the PAH feature emission map and the $\mathrm{H} \alpha$ image of Lehnert \& Heckman (1996) is shown in Fig. 8. That figure clearly shows that the north and south tails seen in the PAH emission trace out the edges of both the approaching (southwest) and receding (northeast) supernovae-driven $\mathrm{H} \alpha$ shells. A less clear correspondence exists with the eastern tail. The appearance of faint
PAH features and their correlation with the shell-like appearance of the $\mathrm{H} \alpha$ emission suggests that extended morphology of the PAH emission may be related to the outflowing wind.

Strickland et al. (2000) and Weaver et al. (2002) have presented Chandra observations of the core of NGC 253. Strickland et al. (2000) find that the soft X-ray emission in the nucleus is edge-brightened with a similar morphology to that seen in $\mathrm{H} \alpha$ while Weaver et al. (2002) find that the hard $\mathrm{X}$-ray $(2.0-8.0 \mathrm{keV})$ is confined to a $5^{\prime \prime}$ region coincident with the radio nucleus. This relatively weak hard X-ray emission is dominated by 3 central point sources with extended 


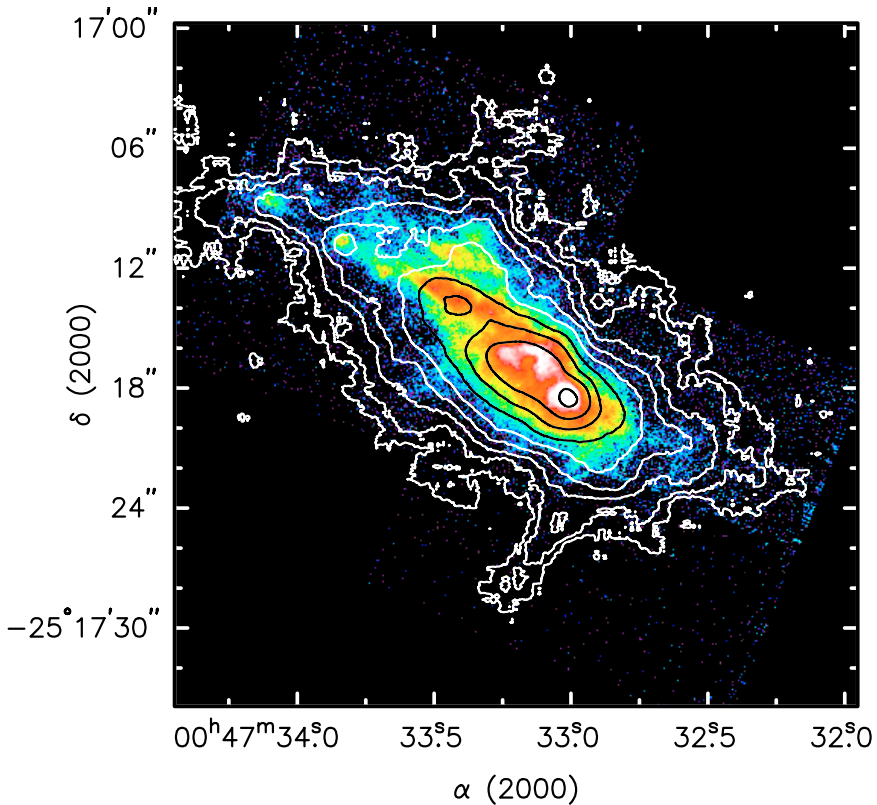

Fig. 5. Logarithmic display of the Paschen $\alpha$ emission from NGC 253 (Alonso-Herrero et al. 2003) with PAH feature emission contours superposed.
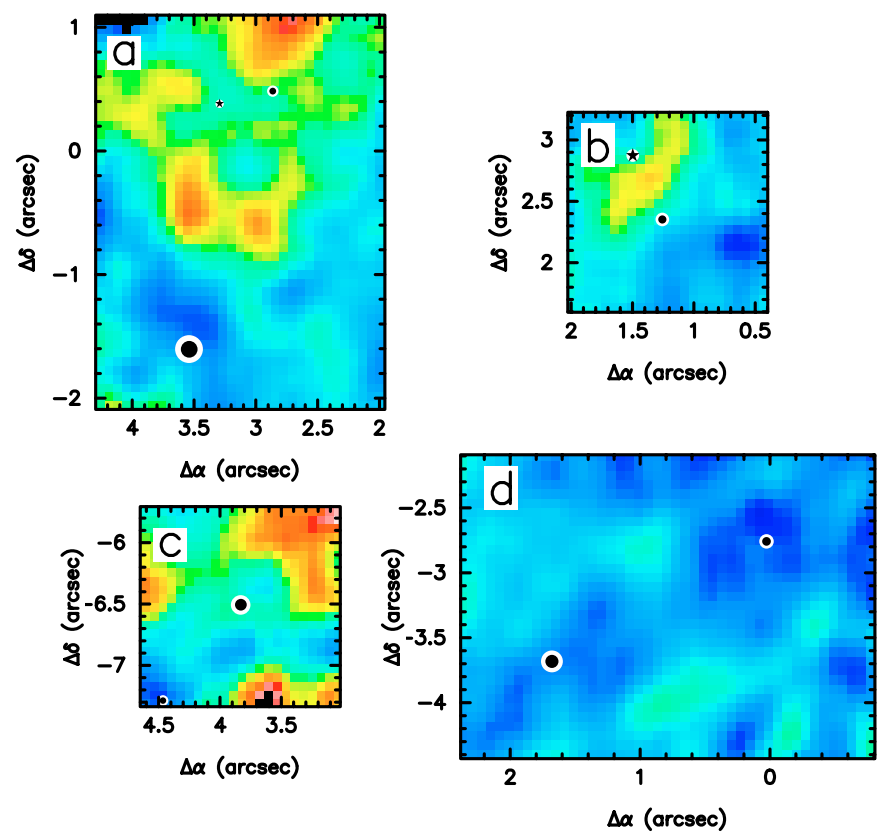

Fig. 6. Blow-ups of regions a-d in Fig. 4 (the symbols and colour scale are the same as in that figure). The labelling on the $x$ - and $y$-axes are offsets relative to the position of the nucleus. In each case the $K$-band knot lies at a position of low feature-to-continuum ratio.

emission. Neither the $3 \mu \mathrm{m}$ continuum, nor the PAH feature emission, nor the feature-to-continuum ratio map show any spatial correlation (or anti-correlation) with the position of the hard X-ray emission. Both the correlation with the soft X-ray emission and the lack of correlation with the hard X-ray emission suggests either that there is some material which shields the PAH molecules from the hard X-rays but not from either the ionising emission from the young star clusters or the
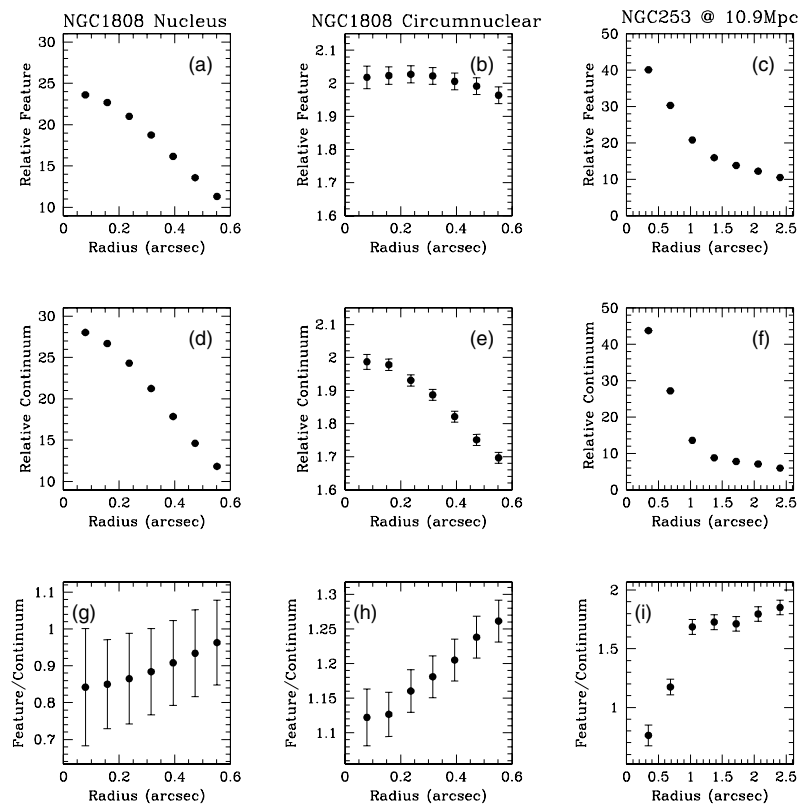

Fig. 7. Radial average plots of the continuum-subtracted PAH feature emission (top row (panels a)-c)), $3 \mu \mathrm{m}$ continuum (middle row (panels d)-f))), and feature-to-continuum ratio (bottom row (panels $\mathbf{g})-\mathbf{i})$ )). The apertures are centred on the nucleus of NGC 1808, the 15 circumnuclear $K$-band knots in NGC 1808, and the bright IR spot in NGC 253 in the first through third columns, respectively. See text for further details.

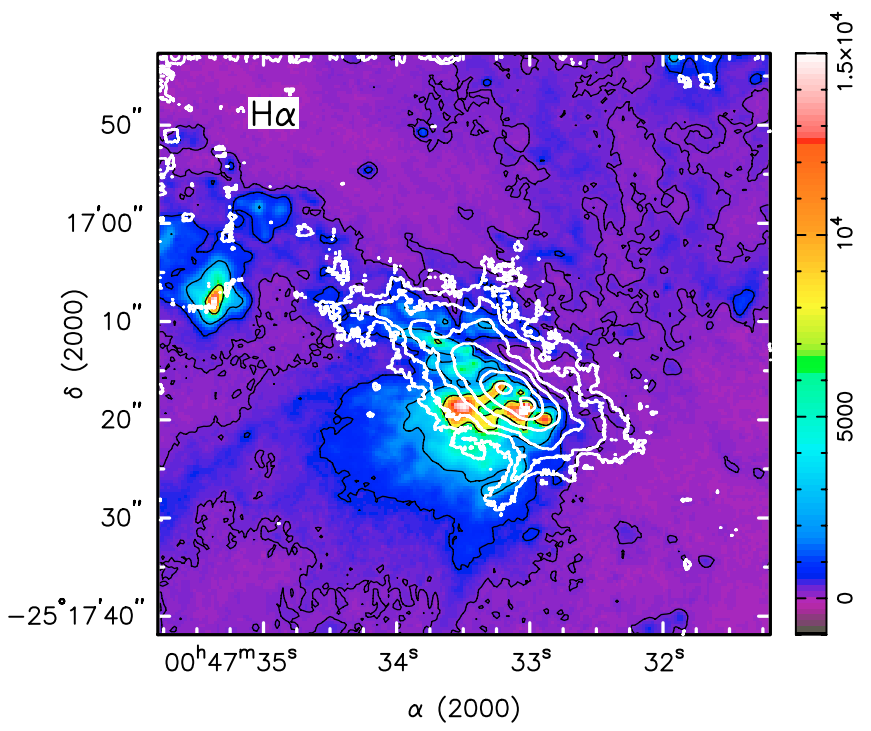

Fig. 8. H $\alpha$ emission from NGC 253 from Lehnert \& Heckman (1996) with white PAH emission contours superposed.

mechanical effects of the outflowing wind, or that the hard Xray emission is too weak to have an impact on the $\mathrm{PAH}-$ feature emitters. Moreover, that there is no direct correlation or anticorrelation with the hard X-ray emission emphasises that the degree to which PAH feature emission traces starburst versus AGN activity is perhaps more complicated than originally perceived. What is required to address this issue more completely are observations of a broader sample of sources covering a wide 
range of star formation rates, AGN activity and/or X-ray luminosity, and metallicity, as discussed in Sect. 1.

\subsection{The bright IR peak in NGC 253}

The nature of the observed clumpy structure seen in the nuclear region of NGC 253 has been addressed by many authors. Sams et al. (1994) used the observed NIR colours to argue that the structure seen was a direct result of patchy extinction which lets a smooth stellar continuum shine through to varying degrees. However, as Forbes et al. (2000) point out, this cannot be the whole picture owing to the organised pattern of optical and NIR knots surrounding the position of the radio nucleus (Turner \& Ho 1985; Antonucci \& Ulvestad 1988; Ulvestad \& Antonucci 1991, 1994, 1997). Based on data ranging from the mid-IR to the optical, several authors have argued that the brightest of these observed extra-nuclear IR emission knots is the location of a young $\left(10^{6}-10^{7}\right.$ year old) (super) star cluster (Watson et al. 1996; Böker et al. 1998; Keto et al. 1999; Forbes et al. 2000). Argument against this being the case comes from the earlier study of the PAH emission from NGC 253 made by Kalas \& Wynn-Williams (1994).

In discussing the nature of the brightest IR feature in the nuclear region of NGC 253 Kalas and Wynn-Williams argue against it being a young cluster of stars based on two arguments. First, their observed $3.28 \mu \mathrm{m}$ PAH emission is particularly weak (relative to the underlying continuum) at that position $^{5}$. A concentration of young stars, they reason, should result in strong PAH emission from the PDRs at the edges of the associated HII regions. Second, the expected radio continuum emission from the corresponding HII regions is not seen. They speculate that this source (designated as Peak 1) might be a dust-enshrouded supernova remnant. Although they find no compelling evidence for expected MIR variability, they do state that future changes could be detected.

Our higher resolution ISAAC observations obviously can shed no light on the lack of radio continuum emis$\operatorname{sion}^{6}$, however our data do also show a minimum of the feature-to-continuum ratio at the position the IR peak (Fig. 3). The time between our observations and those of Kalas and Wynn-Williams is almost exactly 10 years, well-matched to the relevant timescales of an evolving supernova remnant (Kalas \& Wynn-Williams 1994). We have measured the mean feature-to-continuum values over $0.4^{\prime \prime}$ apertures at the positions of their Peaks 1 and 2 and find values of 0.70 and 2.1, respectively. Both of these values are fully consistent with

\footnotetext{
5 Kalas and Wynn-Williams use the mean of the emission at $3.18 \mu \mathrm{m}$ and $3.38 \mu \mathrm{m}$ to represent the continuum underlying the PAH feature emission at $3.28 \mu \mathrm{m}$. As stated in Sect. 3, the emission at $3.38 \mu \mathrm{m}$ is contaminated by a weaker PAH feature at $3.4 \mu \mathrm{m}$ (Sturm et al. 2000, and references therein), resulting in an overestimate of the continuum. Nevertheless if one uses their tabulated $3.18 \mu \mathrm{m}$ measurement as the continuum the feature-to-continuum ratio for their Peak 1 (0.98) is still low relative to that of their Peak 2 (2.13).

6 Forbes et al. (2000) suggest that the predicted value for radio continuum flux is overestimated as a result of the presence of dense gas in the region (Paglione et al. 1995).
}

those values measured by Kalas and Wynn-Williams, implying no temporal variation over a ten year baseline.

This is strong evidence against this source being an evolving supernova remnant, and we thus also favour the (super) star cluster nature of this feature. Moreover, the lower feature-to-continuum ratio at the position of a (super) star cluster in this galaxy is consistent with what we find in the case of NGC 1808 (Sect. 4.3)

\subsection{The nucleus of NGC 253}

That the nucleus itself contains an AGN is suggested by numerous observations. A-array VLA observations at $1.3 \mathrm{~cm}$ reveal an unresolved source of high brightness temperature $\left(T_{\mathrm{b}}>\right.$ $20000 \mathrm{~K}$ at $1.3 \mathrm{~cm}$ ) with a flat spectral index between 2 and $6 \mathrm{~cm}$, indicative of either an AGN or a very compact supernova remnant (Ulvestad \& Antonucci 1997). However, the lack of detected source variability at radio wavelengths over 10 year timescales implies that if it is a SNR it is likely not expanding (Ulvestad \& Antonucci 1997). Further, radio observations of the nucleus have detected both $\mathrm{H}_{2} \mathrm{O}$ maser emission (Nakai et al. 1995) and broad $\left(\Delta V \sim 200 \mathrm{~km} \mathrm{~s}^{-1}\right) \mathrm{H} 92 \alpha$ and $\mathrm{H} 75 \alpha$ recombination lines (Mohan et al. 2002). Although the latter can be interpreted in the context of a young compact stellar cluster Mohan et al. favour a low luminosity AGN model in light of the radio continuum observations of Turner \& Ho (1985) and Ulvestad \& Antonucci (1997). In addition, Weaver et al. (2002) have obtained Chandra observations of NGC 253 which show a hard X-ray source at the position of the nucleus, with an observed $2-10 \mathrm{keV}$ luminosity $\geq 10^{39} \mathrm{ergs} \mathrm{s}^{-1}$, viewed through a dusty medium with a column density $N_{\mathrm{H}} \sim 2 \times$ $10^{23} \mathrm{~cm}^{-1}$. Making the assumption that this source is analogous to Seyfert 2 galaxies with buried nuclei, they estimate the intrinsic X-ray luminosity to be at least $\sim 10^{41} \mathrm{ergs} \mathrm{s}^{-1}$. The fact that our PAH observations show nothing unusual at that position (Fig. 3) may then set a lower limit to the X-ray luminosity required to influence $\mathrm{PAH}$ feature emission.

\section{Discussion}

\subsection{Excitation of PAHs in starbursts}

On both the large scale (Sect. 4.2) and smaller scales (Sect. 4.3) we observe decreased PAH feature-to-continuum ratios, despite the fact that the PAH emission itself is not low. In the absence of a more direct tracer of star formation at sufficiently high spatial resolution, we use the fact that the morphology of the $3.3 \mu \mathrm{m}$ continuum emission and the $\mathrm{Pa} \alpha$ emission in NGC 253 are very similar at least in the high surface brightness inner regions (Fig. 9). At lower surface brightnesses the similarity is less compelling. Thus we take the high surface brightness $3.3 \mu \mathrm{m}$ continuum emission as a surrogate for a starburst UV radiation field in both of our targets.

This can also be justified on the ground that we are primarily restricting ourselves to a discussion of the bright IR peak in NGC 253 as well as the locations of star clusters in NGC 1808. In both cases the star formation has occurred in a burst less 
than $\sim 10^{7}$ yrs old (NGC 253: Watson et al. 1996; Böker et al. 1998; Keto et al. 1999; Forbes et al. 2000; NGC 1808: TGSE96). Indeed, it is the relative youth of the star formation in the clusters of NGC 1808 that makes it possible to identify them in high resolution $K$-band observations. Starburst99 modelling of star clusters like those in NGC 1808 (Leitherer et al. 1999; TGSE96) illustrates that the $3 \mu$ m continuum emission closely tracks that in the $K$-band over the lifetime of a starburst (always within a factor of two). Further, for ages of $\sim 10^{7}$ yrs the luminosity ratio of Lyman continuum to $K$-band (hence also to the $3 \mu \mathrm{m}$ continuum) is still high. Thus, for such young clusters only the $3 \mu \mathrm{m}$ continuum emission can be used as an alternate, though still not perfect, tracer of star formation (Fig. 9).

Since the central starbursting regions in our targets are the very regions where the starburst UV flux is high, the fact that it is there where we observe a low feature-to-continuum ratio could imply that the smoothly distributed, older, circumnuclear star formation has induced a depletion in the number of PAH molecules in the circumnuclear environments in these galaxies as a result of mechanical energy input through stellar winds and supernovae.

An alternate scenario is suggested by the modelling work of Allain et al. (1996a) and Allamandola et al. (1999). Allain et al. have shown that most often the result of the absorption of a UV photon with energies between about 10 and $13.6 \mathrm{eV}$ by a PAH molecule is the ionisation of the molecule, rather than the emission of an IR photon. It is the lower energy photons which are chiefly responsible for the excitation of the $\mathrm{PAH}$ molecules which results in IR emission. Therefore, in regions of star-formation-driven UV flux it is anticipated that the PAHs are predominantly ionised (Allamandola et al. 1999). Further, Allamandola et al. have shown through modelling of PAH absorption spectra that the intensity of the $3.3 \mu \mathrm{m}$ PAH emission feature strongly decreases with respect to features at longer wavelengths, especially that at $7.7 \mu \mathrm{m}$, when PAH molecules become ionised.

Still another process by which the feature-to-continuum ratio could be depressed near regions of young starburst activity is the simple destruction of PAH molecules altogether. Allain et al. (1996b) have shown that PAH molecules which are already either ionised or even partially dehydrogenated have photodissociation rates far in excess of those of the corresponding neutral species. This is consistent with the result of Spoon (2003) who finds that PAH emission better traces B stars than sites of massive star formation.

In spite of the fact that the UV flux must increase at the positions of the $K$-band knots in NGC 1808 , the PAH feature emission on average is essentially flat at those positions (Fig. 7b), resulting in a feature-to-continuum ratio which shows a minimum there. However, the fact that the PAH feature emission does not show an enhancement at those positions, coupled with the fact that the knots are relatively young (of order $10^{7} \mathrm{yr}$ old (TGSE96)), argues in favour of the PAH molecules either being ionised or destroyed at those positions. The nucleus of NGC 1808, on the other hand, shows a peak in both the PAH feature emission and the adjacent continuum, resulting in a feature-to-continuum ratio which is consistent with being flat or only very slightly rising with distance from the nucleus. Thus, any modifications of the PAH emission efficiency or number density must not play a dominant role at that position, likely as a result of the older (200 Myr (TGSE96)) star formation at that position. The mechanical energy input to the ISM from this older star formation may explain a slightly broader PAH feature radial profile than that of the continuum emission.

That PAH destruction plays a particularly important role in the observed $3.3 \mu \mathrm{m}$ feature-to-continuum ratio at the position of the IR peak in NGC 253 is perhaps even more difficult to discount. As we already discussed in Sect. 4.5, we favour the super star cluster interpretation of the IR peak in NGC 253. The inferred mass of the cluster, $10^{5} M_{\odot}$ (Forbes et al. 2000), is consistent with that inferred for the circumnuclear knots in NGC 1808 (TGSE96). The age of the star cluster in NGC 253 has been estimated by a number of authors (Watson et al. 1996; Keto et al. 1999; Forbes et al. 2000), all of which agree that it must be very young $(<50 \mathrm{Myr}$, or perhaps even younger than $1 \mathrm{Myr}$ ), with some 1000 or more $\mathrm{O}$ stars still on the main sequence. In addition, the cluster is much more compact than those seen in the circumnuclear region of NGC 1808 (Fig. 7). Although to our knowledge no high spatial resolution observations of cold molecular gas exist for either target considered here, the $\mathrm{H}_{2}$ observations of NGC 253 by Sugai et al. (2003) show that the there is a peak in the hot molecular component at the location of the IR peak. That this gas is spatially coincident with the region of PAH emission is shown in Fig. 10, implying that there exist PAH molecules associated with the warm molecular component of the ISM. Further, it is quite likely given the relative youth and compactness of the cluster in NGC 253 that there remains a large concentration of dusty, PAH-bearing, cold molecular gas at that location. However, of the three cases presented in Fig. 7, it is the bright IR peak in NGC 253 which shows the lowest feature-to-continuum ratio. Thus, the $3.3 \mu \mathrm{m}$ PAH feature emission may be strong because of the still relatively large concentration of viable $3.3 \mu \mathrm{m}$ feature-emitting PAH molecules, but the ratio low owing to the relative inefficiency of emission at that wavelength.

Although from our present data we have no way to disentangle the effects of photoionisation and photodestruction, ISOCAM observations of NGC 253, albeit at much lower spatial resolution, have revealed variations in the circumnuclear PAH $8.6 \mu \mathrm{m} / \mathrm{PAH} 11.3 \mu \mathrm{m}$ ratio which may indicate that photoionisation is playing a role (Förster Schreiber et al. 2003, and references therein). There is an observation which could shed further light on the matter. The photoionisation of PAH molecules also results in a strong enhancement of $7.7 \mu \mathrm{m}$ PAH feature emission relative to the feature at $3.3 \mu \mathrm{m}$. Photodissociation, on the other hand, results in both lines being suppressed. High spatial resolution observations of the $7.7 \mu \mathrm{m}$ PAH feature, especially when compared to the $3.3 \mu \mathrm{m}$ continuum, would allow us to discern which of these two mechanisms is the dominant one.

Further, high spatial resolution imaging observations of an IR tracer of star formation, such as $\operatorname{Br} \gamma$ or $\operatorname{Pa} \alpha$, are required to probe the dusty star-forming locations and to provide a more direct measure of the UV radiation intensity available to excite, ionise, or destroy the PAH molecules. 


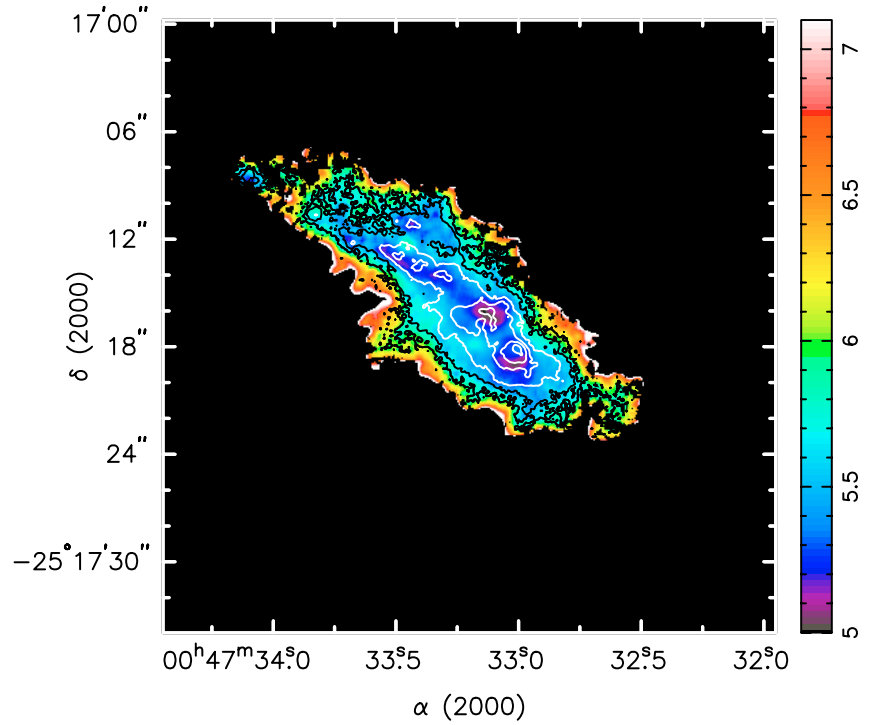

Fig. 9. Logarithm of the ratio of the $3.3 \mu \mathrm{m}$ continuum emission to the Paschen $\alpha$ emission in NGC 253 in color with contours of the $\log$ of the Paschen $\alpha$ emission superposed. The Paschen $\alpha$ data has been convolved to the spatial resolution of the $3.3 \mu \mathrm{m}$ data. As the $3.3 \mu \mathrm{m}$ continuum data is not flux calibrated, the contours (with values of $-13,-12, \ldots,-8)$ are relative. We find that the ratio of $(3.3 \mu \mathrm{m}$ continuum/Paschen $\alpha$ ) is much smoother in the central regions than is the Paschen $\alpha$ emission itself. While this supports our contention that the $3 \mu \mathrm{m}$ continuum is a reasonable surrogate for the ionising flux in young star forming regions the robustness of this supporting evidence is limited perhaps by the dynamic range of the ISAAC data (which is lower than that of the NICMOS data).

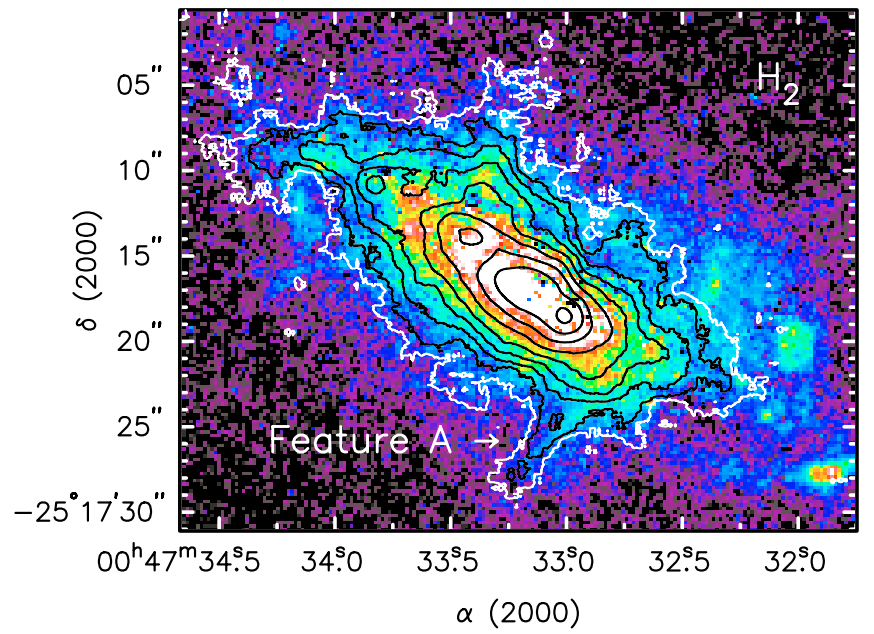

Fig. 10. $\mathrm{H}_{2} v=1-0 \mathrm{~S}(1)$ emission from NGC 253 from Sugai et al. (2003) with PAH emission contours superposed. The southern tail is designated as Feature A by Sugai et al.

\subsection{Implications for superwinds}

The relationships between the various phases of gas - from the hot X-ray emitting gas down through the dust emission - can be used to place constraints of the source of the observed wind material.

Observing extended PAH emission along the minor axis of NGC 253 suggests that the outflowing gas contains molecular

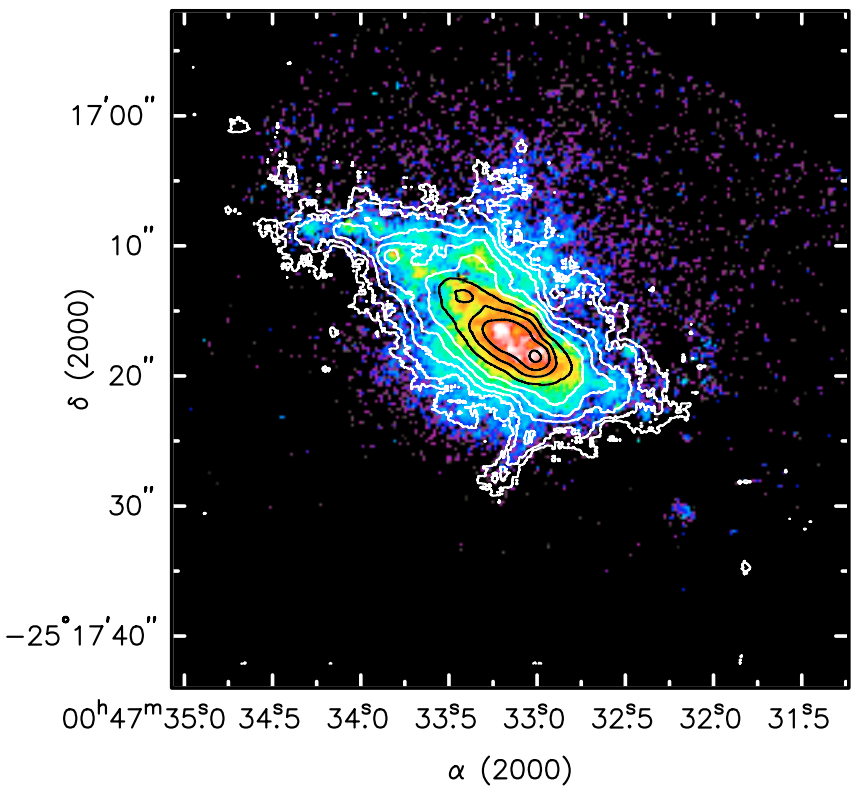

Fig. 11. [FeII $] 1.644 \mu \mathrm{m}$ emission from NGC 253 from Alonso-Herrero et al. (2003) with PAH emission contours superposed.

gas and/or small grains. Other observations also suggested this is the case. Sugai et al. (2003) have shown that narrow dust lanes as seen in HST WFPC2 archival data in the $F 814 \mathrm{~W}$ band align very well with the edge of the X-ray plasma bubble. In addition, Heckman et al. (2000) argued that dust grains must survive in the wind to keep the high velocity $\mathrm{NaD}$ absorption observed in starbursts with superwinds from being ionised ( $\mathrm{Na}$ has an ionisation potential low enough to be ionised by soft, non-ionising UV photons). More direct evidence for dust survival in winds comes from ISOPHOT observations which show extended 120 and $180 \mu \mathrm{m}$ emission along the minor axis of NGC 253 (Radovich et al. 2001). This being the case, it seems most likely that the gas originated in the plane or within a few disc scale heights of the plane of NGC 253 to explain a significant molecular or dust content. Cooling gas in the wind material itself can be eliminated since it is difficult to understand how such molecules and small grains can form in the cooling region of the wind. Although clouds in the halo or at many disc scale heights shock-heated by the wind have been observed (in M 82 for example, Lehnert et al. 1999) the morphology and relatively strong PAH emission argues against such a hypothesis in this case since again how do the carriers of the PAH get there in the first place? Thus the only reasonable hypothesis that would explain the presence of PAHs on the one hand and the morphology of the emission on the other is that the bubble/shell-like morphology of both the $\mathrm{H} \alpha$, soft X-ray, and PAH emission represents entrained and swept-up material from close into the nucleus and at less than a few disc scale-heights and that the wind must be heavily mass-loaded (in agreement with other arguments; see Moran et al. 1999 for example). But how was the entrained/swept-up gas able to have its PAH emitting molecules survive the intense nuclear UV/Xray field, the acceleration due to the shocks, and collisions with the atomic nuclei and fast moving electrons? Since the exact sources of the PAH emission features are not known precisely, 
it is difficult to propose a quantitative solution. However, clues may be provided by the results of Sugai et al. (2003). These authors have recently reported on UKIRT and HST observations of extended plumes of $\mathrm{H}_{2}$ emission from the nuclear region of NGC 253. The location and spatial extent of the $\mathrm{H}_{2}$ plumes, especially their Feature A, bears a striking similarity to those observed in PAH emission (Fig. 10).

In discussing the origin of the $\mathrm{H}_{2}$ gas, Sugai et al. come to the conclusion that either the gas is blown out by the wind, or pushed to the side by the expanding bubble by the hot plasma seen in X-rays, similar to what we have posited above for the PAH molecules. In addition, although the issue of the excitation of the $\mathrm{H}_{2}$ in these plumes remains unresolved, Sugai et al. do find that the X-rays from the bubble itself are insufficient, as is the UV flux from the starburst. Thus it may be that if the $\mathrm{H}_{2}$ gas and the PAH molecules share a common location the threat posed by these two wavelengths of radiation to the PAH molecules is not substantial. Finally, although Sugai et al. cannot rule out excitation of the $\mathrm{H}_{2}$ through X-rays from the nucleus itself, they favour the model in which shock excitation is the dominant mechanism. Support for the presence of shocks in this region comes also from the excellent morphological agreement between the extended PAH feature tails/countertails and the extended [FeII] emission (Alonso-Herrero et al. 2003, Fig. 11). If the shocks responsible for the $\mathrm{H}_{2}$ excitation in NGC 253 are similar in strength to those responsible for the bulk of the $\mathrm{H}_{2}$ excitation in Mrk 266 (Davies et al. 2000) they would also be insufficiently strong to destroy the PAH molecules (Flower \& Pineau des Forêts 2003). As an additional note, such a shock could actually cause a buildup of PAHs at that position (Jones et al. 1996), though for that to be the case it would require even heavier dust particles to have been carried there.

Not only does the survivability of the molecular gas pose an interesting puzzle, but in what phase of the ISM it originates and how it gets into the halo are also puzzling. The surface brightness of the $\mathrm{H}_{2}$ emission is consistent with molecular $\mathrm{H}_{2}$ emission from shocks with velocities of $10-20 \mathrm{~km} \mathrm{~s}^{-1}$ (Sugai et al. 2003). Balancing the momentum across the shock implies that the speed of the shock driven into the cloud will be related to the superwind velocity by the inverse of the square root of the cloud-wind density contrast. The kinematics of the optical emission line gas and the properties of the soft X-ray emission suggest minimum outflow velocities of roughly $900 \mathrm{~km} \mathrm{~s}^{-1}$ (Strickland et al. 2000). Hydrodynamical wind models suggest hot wind densities of of about $10^{-3}-10^{-2} \mathrm{~cm}^{-3}$ (Strickland \& Stevens 2000, see also Chevalier \& Clegg 1985). Taking a shock velocity of $10 \mathrm{~km} \mathrm{~s}^{-1}$ in the molecular cloud where the $\mathrm{H}_{2} / \mathrm{PAH}$ originate and assuming (for convenience) an outflow velocity of $v_{\text {outflow }}=$ $1000 \mathrm{~km} \mathrm{~s}^{-1}$, this would suggest a density of the pre-shocked clouds of $10 v_{\text {outflow,1000 }}^{2} n_{\text {wind,-3 }} \mathrm{cm}^{-3}$ (where $v_{\text {outflow,1000 }}=$ $v_{\text {outflow }} / 1000 \mathrm{~km} \mathrm{~s}^{-1}$, and $n_{\text {wind,-3 }}=n_{\text {wind }} / 10^{-3} \mathrm{~cm}^{-3}$ ). Clouds become self shielding at $A_{\mathrm{V}} \approx 1$ or column densities about $10^{21} \mathrm{~cm}^{-2}$ (Burton et al. 1990). This would suggest clouds of approximately $30 \mathrm{pc}$ or larger. Heckman et al. (2000) estimated similar column densities to explain the high optical depth of the
$\mathrm{NaD}$ absorption lines in a sample of nearby starburst galaxies. Since dust is required to shield neutral $\mathrm{Na}$ (which has an ionization potential of $5.4 \mathrm{eV}$, roughly similar to the energy required to dissociate $\mathrm{H}_{2}$ and ionize/destroy PAHs), it seem like a reasonable hypothesis that the $\mathrm{NaD}$ absorption, the $\mathrm{H}_{2}$ emission, and $\mathrm{PAH}$ feature emission all originate from the same material with the PAHs and larger dust grains providing the shielding opacity for the Na. As found for the $\mathrm{NaD}$ lines in Heckman et al., this hypthesis would also predict outflow velocities of several $100 \mathrm{~km} \mathrm{~s}^{-1}$ for $\mathrm{H}_{2}$ and PAH emission which would be interesting to test. This would also provide more than enough time for the carriers of PAH to reach the heights above the disk as we have observed.

However, as the present data are still rather shallow (though deeper than those of Kalas and Wynn-Williams who did not detect this extended emission), we cannot say for certain what the overall relation is between the PAH molecules, the outflowing wind and various phases of the ISM. Deeper exposures reaching fainter gas out in the halo are needed to address that issue. For the present, let us simply say that we see tantalising evidence that PAH molecules can survive in environments as hostile as winds and that their existence provides strong support that winds are heavily mass-loaded by the ambient ISM and that material from within several scale heights of the disc are driven out into the halo. More fundamentally, it would be very interesting to investigate the dynamics of the PAH emitting gas to see if it is participating in the outflow seen in $\mathrm{H} \alpha$ and perhaps escaping the galaxian potential and influencing the dust grain size distribution in the intergalactic medium. The properties of dust in the intergalactic medium are of obvious cosmological interest (e.g. Leibundgut 2001).

\section{Conclusions}

We have presented first narrowband observations of $3.3 \mu \mathrm{m}$ PAH feature emission in the two nearby starburst galaxies NGC 253 and NGC 1808. From these observations we find that the PAH emission is seen to peak in the inner, intensely starbursting regions of these galaxies. This could be interpreted as PAH feature emission directly tracing starburst regions of galaxies. However, when we look in detail at the distribution of PAH emission with respect to the locations of known recent star formation, in the form of radio supernova remnants and $K$-band knots, we find no spatial coincidence, either positive or negative. This is consistent with the finding of Spoon (2003) that the PAH emission may better trace the general B star population than the sites of massive recent star formation. Moreover, this is perhaps analogous to the contribution of the diffuse ionised gas to the $\mathrm{H} \alpha$ budget seen in spiral and starburst galaxies (e.g. Walterbos \& Braun 1994; Wang et al. 1998).

Although we see no correlation between the PAH feature emission and the location of sites of recent star formation, we do see a decrease in the feature-to-continuum at these sites. We take this to imply a decrease in the efficiency of PAH emission induced by the star formation itself. We have discussed three models to account for this: mechanical energy input into the ISM, photoionisation of the PAH molecules, and 
photodissociation of the PAH molecules. The first of these mechanisms may explain the observations of the nucleus of NGC 1808, as star formation at that location is older (TGSE96). The latter two mechanisms may be responsible for the decrease in the feature-to-continuum ratio seen at the locations of the circumnuclear starburst sites in NGC 1808 and the bright IR peak in NGC 253. The present data cannot distinguish between photoionisation and photodissociation, and high spatial resolution $7.7 \mu \mathrm{m}$ observations would be useful in that context. Low spatial resolution ISOCAM observations of NGC 253 may indicate that PAH photoionisation does play a role. In summary, we find that the ratio of PAH feature emission to stellar continuum varies with local conditions inside a starburst. This variation introduces an uncertainty in quantitatively using the PAH features to decompose the starburst and AGN activity of galaxies.

We find no temporal variation in the $\mathrm{PAH}$ featureto-continuum ratio from the bright IR peak in NGC 253 when comparing our observations to those of Kalas \& Wynn-Williams (1994). Such variation would be predicted if this source was a heavily dust-enshrouded SNR. This lack of observed variation is then compelling evidence against that being the nature of that source and instead we favour the very young super star cluster nature of this feature.

Finally, we presented the first observations of PAH molecules in a starburst-driven galactic superwind (NGC 253). We argue that the PAHs in that wind must have originated in or within a few scale-heights of the disk, and are being driven out into the halo of the system. The shocks associated with this wind are sufficient to excite the co-extensive $\mathrm{H}_{2}$ molecules (Sugai et al. 2003), but insufficient to destroy the PAH molecules. Indeed, the shocks may be responsible for the PAH molecules in the first place, as modelled by Jones et al. (1996), though the required ingredient for that would be even heavier dust particles. Whether the PAH molecules, or the even heavier dust particles, entrained in the wind can escape the galaxian potential is a very interesting question, though one which is technically challenging to answer.

Acknowledgements. We wish to thank Sabine Mengel and Fernando Comerón for stimulating conversations. In addition, we would like to thank Almudena Alonso-Herrero for making her data available to us in electronic form. Finally, we thank the anonymous referee for comments which improved the text of this paper. This research has made use of the NASA/IPAC Extragalactic Database (NED) which is operated by the Jet Propulsion Laboratory, California Institute of Technology, under contract with the National Aeronautics and Space Administration.

\section{References}

Allain, T., Leach, S., \& Sedlmayr, E. 1996a, A\&A, 305, 602 Allain, T., Leach, S., \& Sedlmayr, E. 1996b, A\&A, 305, 616 Allamandola, L. J., Hudgins, D. M., \& Sandford, S. A. 1999, ApJ, 511, L115

Alonso-Herrero, A., Rieke, G. H., Rieke, M. J., et al. 2003, AJ, 125, 1210

Antonucci, R. R. J., \& Ulvestad, J. S. 1988, ApJ, 330, L97

Armus, L., Charmandaris, V., Spoon, H. W. W., et al. 2004, ApJS, 154, 178
Awaki, H., Ueno, S., Koyama, K., et al. 1996, PASJ, 48, 409

Böker, T., Krabbe, A., \& Storey, J. W. V. 1998, ApJ, 498, L115

Boulanger, F., Beichman, C., Desert, F. X., et al. 1988, ApJ, 332, 328

Burton, M. G., Hollenbach, D. J., \& Tielens, A. G. G. M. 1990, ApJ, 365,620

Chevalier, R. A., \& Clegg, A. W. 1985, Nature, 317, 44

Clavel, J., Schulz, B., Altieri, B., et al. 2000, A\&A, 357, 839

Collison, P. M., Saikia, D. J., Pedlar, A., et al. 1994, MNRAS, 268, 203

Contursi, A., Lequeux, J., Cesarsky, D., et al. 2000, A\&A, 362, 310

Davies, R., Ward, M., \& Sugai, H. 2000, ApJ, 535, 735

Devost, D., Brandl, B. R., Armus, L., et al. 2004, ApJS, 154, 242

Flower, D. R., \& Pineau des Forêts, G. 2003, MNRAS, 343, 390

Förster Schreiber, N. M., Sauvage, M., Charmandaris, V., et al. 2003, A\&A, 399, 833

Forbes, D. A., Polehampton, E., Stevens, I. R., et al. 2000, MNRAS, 312,689

Genzel, R., Lutz, D., Sturm, E., et al. 1998, ApJ, 498, 579

Harvey, V. I., Satyapal, S., Luhman, M. L., et al. 1999, FarInfrared Spectroscopy and Near-Infrared Imaging of Mrk 231, in The Universe as Seen by ISO, ed. P. Cox, \& M. F. Kessler, ESA-SP427, 889

Heckman, T. M., Lehnert, M. D., Strickland, D. K., et al. 2000, ApJS, 129,493

Helou, G., Lu, Nanyao Y., Werner, M. W., et al. 2000, ApJ, 532, L21

Houck, J. R., Charmandaris, V., Brandl, B. R., et al. 2004, ApJS, 154, 211

Johnson, K. E., Kobulnicky, H. A., Massey, P., et al. 2001, ApJ, 559, 864

Jones, A. P., Tielens, A. G. G. M., \& Hollenbach, D. J. 1996, ApJ, 469,740

Kalas, P., \& Wynn-Williams, C. G. 1994, ApJ, 434, 546

Keto, E., Ball, R., Arens, J., et al. 1993, ApJ, 413, L23

Keto, E., Hora, J. L., Fazio, et al. 1999, ApJ, 518, 183

Kotilainen, J. K., Forbes, D. A., Moorwood, A. F. M., et al. 1996, A\&A, 313, 771

Krabbe, A., Sternberg, A., \& Genzel, R. 1994, ApJ, 425, 72

Laurent, O., Mirabel, I. F., Charmandaris, V., et al. 2000, A\&A, 359, 887

Laurent, O., Tran, D., Lutz, D., et al. 2001, ApSSS, 277, 101

Le Floc'h, E., Mirabel, I. F., Laurent, O., et al. 2001, A\&A, 367, 487

Lehnert, M. D., \& Heckman, T. M. 1996, ApJ, 462, 651

Lehnert, M. D., Heckman, T. M., \& Weaver, K. A. 1999, ApJ, 523, 575

Leibundgut, B. 2001, ARA\&A, 39, 67

Leitherer, C., Schaerer, D., Goldader, J. D., et al. 1999, ApJS, 123, 3

Luhman, M. L., Satyapal, S., Fischer, J., et al. 2003, ApJ, 594, 758

Lutz, D., Spoon, H. W. W., Rigopoulou, D., et al. 1998, ApJ, 505, L103

Madden, S. C. 2000, NewAR, 44, 249

Mazzarella, J. M., Voit, G. M., Soifer, B. T., et al. 1994, AJ, 107, 1274

Mirabel, I. F., Laurent, O., Sanders, D. B., et al. 1999, A\&A, 341, 667

Mohan, N. R., Anantharamaiah, K. R., \& Goss, W. M. 2002, ApJ, 574, 701

Moorwood, A. F. M. 1986, A\&A, 166, 4

Moorwood, A. F. M. 1999, ISO observations of active galaxies, in The Universe as Seen by ISO, ed. P. Cox, \& M. F. Kessler, ESA-SP427, 825

Moran, E. C., Lehnert, M. D., \& Helfand, D. J. 1999, ApJ, 526, 649

Nakai, N., Inoue, M., Miyazawa, K., et al. 1995, PASJ, 47, 771

Normand, P., Rouan, D., Lacombe, F., et al. 1995, A\&A, 297, 311

Paglione, T. A. D., Tosaki, T., \& Jackson, J. M. 1995, ApJ, 454, L117

Phillips, A. C. 1993, AJ, 105, 486 454, L117 
Pietsch, W., Vogler, A., Klein, U., et al. 2000, A\&A, 360, 24

Piña, R. K., Jones, B., Puetter, R. C., et al. 1992, ApJ, 401, L75

Radovich, M., Kahanpää, J., \& Lemke, D. 2001, A\&A, 377, 73

Rigopoulou, D., Spoon, H. W. W., Genzel, R., et al. 1999, AJ, 118, 2625

Roche, P. F, Aitken, D. K., Smith, C. H., et al. 1991, MNRAS, 248, 606

Saikia, D. J., Unger, S. W., Pedlar, A., et al. 1990, MNRAS, 245, 397

Satyapal, S., Watson, D. M., Pipher, J. L., et al. 1995, ApJ, 448, 611

Sams, B. J., Genzel, R., Eckart, A., et al. 1994, ApJ, 430, L33

Smith, J. D. T., Dale, D. A., Armus, L., et al. 2004, ApJS, 154, 199

Spoon, H. W. W. 2003, Ph.D. Thesis, Rijksuniversiteit Groningen

Spoon, H. W. W., Armus, L., Cami, J., et al. 2004, ApJS, 154, 184

Strickland, D. K., Heckman, T. M., Weaver, K. M., et al. 2000, AJ, 120,2965

Strickland, D. K., Heckman, T. M., Weaver, K. M., et al. 2002, ApJ, 568,689

Strickland, D. K., \& Stevens, I. R. 2000, MNRAS, 314, 511

Sturm, E., Lutz, D., Tran, D., et al. 2000, A\&A, 358, 481
Sugai, H., Davies, R. I., \& Ward, M. J. 2003, ApJ, 584, L9

Tacconi-Garman, L. E., Sternberg, A., \& Eckart, A. 1996, AJ, 112, 918

Tran, Q. D., Lutz, D., Genzel, R., et al. 2001, ApJ, 552, 527

Turner, J. L., \& Ho, P. T. P. 1985, ApJ, 299, L77

Turner, P. C., Forrest, W. J., Pipher, J. L., et al. 1992, ApJ, 393, 648

Ulvestad, J. S., \& Antonucci, R. R. J. 1991, AJ, 102, 875

Ulvestad, J. S., \& Antonucci, R. R. J. 1994, ApJ, 424, 29

Ulvestad, J. S., \& Antonucci, R. R. J. 1997, ApJ, 488, 621

Véron-Cetty, M. P., \& Véron, P. 1985, A\&A, 145, 425

Verstraete, L., Puget, J. L., Falgarone, E., et al. 1996, A\&A, 315, L337

Voit, G. M. 1992, MNRAS, 258, 841

Watson, A. M., Gallagher, J. S. III, Holtzman, J. A., et al. 1996, AJ, 112,534

Wang, J., Heckman, T. M., \& Lehnert, M. D. 1998, ApJ, 509, 93

Walterbos, R. A. M., \& Braun, R. 1994, ApJ, 431, 156

Weaver, K. A., Heckman, T. M., Strickland, D. K., et al. 2002, ApJ, 576, L19 